\title{
Metabolic Control Analysis of Complex Biological Systems
}

\author{
Choamun Yun, Young Kim, Sang Yup Lee and Sunwon Park* \\ Department of Chemical and Biomolecular Engineering, KAIST, Daejeon 305701, Korea \\ *(Tel: 82-42-869-3920; e-mail: sunwon@kaist.ac.kr)
}

\begin{abstract}
A systematic approach is proposed for the metabolic control analysis of various dynamic behaviors in biological systems. The complex kinetic models are reduced by identifying the conservation relations and the time scale analysis. Subsequently, the biological systems are categorized into the three groups: systems with the steady-states, sustained oscillations, or other non-steady states. The sensitivities are measured to calculate the corresponding control coefficients that are defined considering the characteristics of the dynamics of the systems. The proposed approach is illustrated with its application to the dynamic behaviors of a complex biological model.
\end{abstract}

\section{INTRODUCTION}

The newly-developed high-throughput devices have contributed to the recent growing interests in dynamic modelling and analysis of the living systems (Johnson et al., 2004; Villas-Boas et al., 2005), which provide invaluable information for biological research. The main purpose of dynamic analysis is to predict the changes in system behaviours upon given perturbations. Therefore, the sensitivities of a system, i.e., the changes of system responses to the internal or external perturbation, are measured for metabolic control analysis (Kacser and Burns, 1973; Heinrich and Rapoport, 1974a, b). While many models show the stable steady-states of similar characteristics to Teusink model (Teusink et al., 2000), others exhibit periodic phenomena as in yeast glycolytic oscillations (Ghosh and Chance, 1964) or display chaotic behaviours. In this work, the aforementioned behaviours are classified for the dynamic analysis of various biological models.

\section{CONTROL COEFFICIENTS IN METABOLIC CONTROL ANALYSIS}

The changes of network activities to perturbations are quantified by identifying the inherent relationships between the relative changes in the system response, e.g., flux or metabolite concentration, to the perturbed parameter. The relationships inform how the control of fluxes or intermediate concentrations is distributed among the different enzymes in a metabolic pathway. Instead of assuming that flux is controlled by one rate-limiting step, it assumes that it is controlled by a number of parameters (Gunawardena, 2002).

\subsection{Steady-states}

Metabolic control analysis was first developed as a mathematical formalism for the steady-state control and sensitivity analysis of biological systems (Kacser and Burns, 1973). The control coefficients are defined to be the system properties of an enzyme that express how a systemic variable depends on the activity of the enzyme. The flux control coefficient, i.e., the change of steady-state flux of a reaction to the change of the concentration of enzymes or intermediate species, $C_{a}$, can be written as:

$$
C_{a}=\frac{\Delta F_{j}}{\Delta\left[e_{i}\right]}
$$

where $F_{j}$ is the steady-state flux of reaction step $j$, and $\left[e_{i}\right]$, the concentration of the enzyme for the reaction step $i$. Absolute changes of $\left[e_{i}\right]$ and $F_{j}$ depend on their units used to measure them, influencing the magnitude of $\mathrm{Ca}$. Hence, it is preferable to normalize the changes in $\left[e_{i}\right]$ and $F_{j}$, which gives

$$
C=\frac{\Delta F_{j}}{\Delta\left[e_{i}\right]} \frac{\left[e_{i}\right]}{F_{j}}
$$

At the limit of an infinitesimally small changes in $\left[e_{i}\right]$ and $F_{j}$, the flux control coefficient is given by

$$
C_{e_{i}}^{J_{j}}=\left(\frac{d F_{j}}{d\left[e_{i}\right]} \frac{\left[e_{i}\right]}{F_{j}}\right)=\left(\frac{d \ln F_{j}}{d \ln \left[e_{i}\right]}\right)
$$

The larger the flux control coefficient means the greater degree of control an enzyme $e_{i}$ has on the steady-state flux $F_{j}$. For the experimental design, the flux control coefficient is often also defined as

$$
C_{r_{i}}^{F_{j}}=\left(\frac{d F_{j}}{d r_{i}} \frac{r_{i}}{F_{j}}\right)=\left(\frac{d \ln F_{j}}{d \ln r_{i}}\right)
$$

where $r_{i}$ is the rate of reaction step $i$ that changes to the perturbation in the concentration of the enzyme for reaction 
step $i,\left[e_{i}\right]$. The flux control coefficient reveals the relative changes between the steady-state flux and the rate of reaction step $i$.

\subsection{Sustained Oscillation}

A certain biological system returns to the sustained oscillation after a perturbation in the system. Reijenga et al. (2002) defined the response coefficients for the systems with oscillatory behaviors. The changes in the period and the amplitude are measured instead of the steady-state flux. With respect to the change in a parameter, e.g., the concentration of enzyme, $e_{0}$, the changes in the period, $P$, and the amplitude, $K$, are measured as:

$$
\begin{array}{ll}
R_{e}^{P}(t):=\left.\frac{\partial P(t, e)}{\partial e}\right|_{e=e_{0}} & \text { for all } t \geq 0 \\
R_{e}^{K}(t):=\left.\frac{\partial K(t, e)}{\partial e}\right|_{e=e_{0}} & \text { for all } t \geq 0
\end{array}
$$

The period and amplitudes can be measured from the dynamic simulation of the target model. As it takes some time to reach the sustained oscillatory state after a parameter perturbation is made on a system, the early transient part of the time horizon should be eliminated from the time data.

\subsection{Other Non-steady States: Time-varying control coefficients}

For the systems that do not reach the steady-state, the control coefficients are defined by extending the definition of sensitivity functions to time-varying ones (Acerenza et al., 1989; Hu and Yuan, 2006). The coefficients are calculated by assuming that the response of a system is at the pseudosteady-state. Accordingly, it is not the one control coefficient but the trajectories of them that provide the information on its dynamics.

\section{SYSTEMATIC APPROACH FOR METABOLIC CONTROL ANALYSIS}

The current work proposes the systematic approach for classifying the biological systems into three aforementioned control coefficients. As illustrated in Fig. 1, the size of the complex biological systems is firstly reduced by eliminating the singular part of the models. Subsequently, the steadystates are identified and their stabilities are investigated to examine the behaviours about the steady-state. Finally, the control coefficients corresponding to each group of behaviours are obtained.

\subsection{Identification of Conservation (Moiety) Relations}

A set of kinetic equations for a biological system includes the dependent as well as the independent relations. Those dependent relations correspond to the linearly dependent rows in the stoichiometry matrix. The concentration changes of $\mathrm{n}$ biochemical species are equivalent to the rate of $\mathrm{m}$ reactions, multiplied by the matrix of stoichiometric coefficients, $S$.

$\frac{d}{d t} C(t)=S \cdot r(C(t), k) \quad$ for all $t \geq 0$

where $C(t)$ is the vector of concentrations and $k$ is the rate constant. The null space $N$ of stoichiometry matrix $S$, and the reduced matrix $S_{R}$ have the following relationship.

$S=N S_{R}=\left[\begin{array}{c}I \\ M\end{array}\right]$

where $I$ is the identity matrix, and the remaining part is $M$. Accordingly, $C(t)$ is rearranged.

$C(t)=\left[\begin{array}{l}C_{i}(t) \\ C_{d}(t)\end{array}\right]$

$C_{i}$ and $C_{d}$ are the vectors for the concentrations of independent and dependent species, respectively. Substituting (2) into (1),

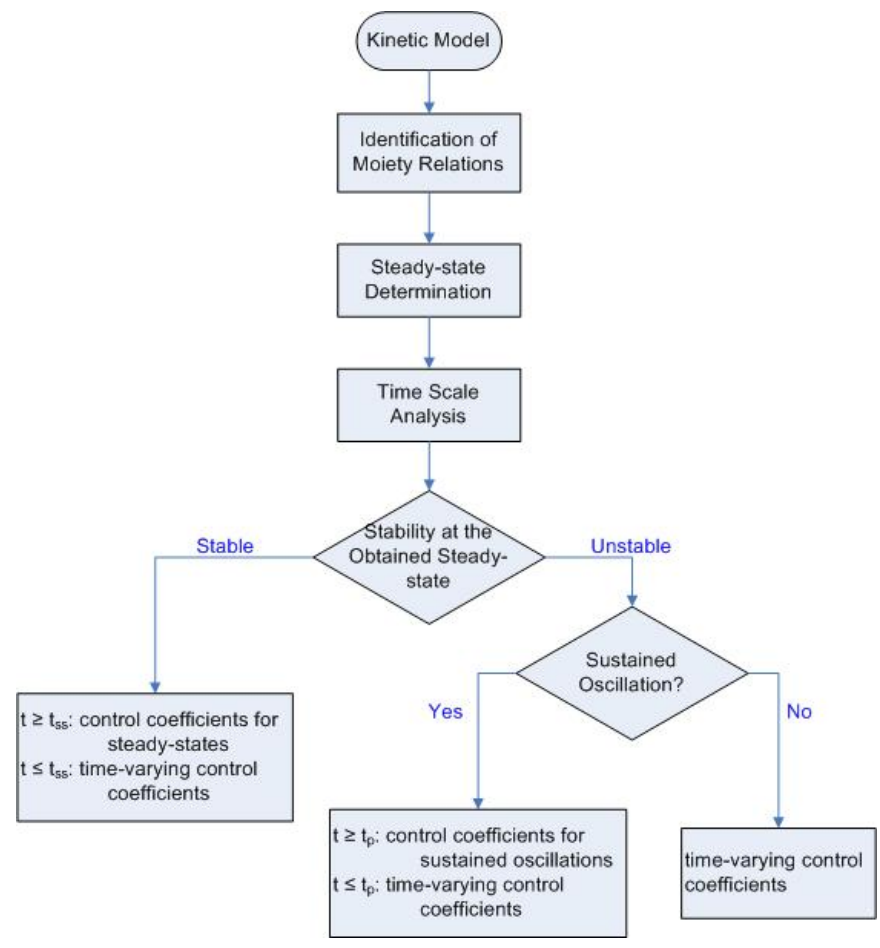

Fig. 1. Flowchart describing the proposed systematic approach for metabolic control analysis of general biological systems. $t_{s s}$ and $t_{p}$ are the time point when systems reach the steady-state or the periodic behaviour, respectively. 


$$
\frac{d}{d t} C(t)=\frac{d}{d t}\left[\begin{array}{l}
C_{i}(t) \\
C_{d}(t)
\end{array}\right]=N S_{R} r(C(t), k) \quad \text { for all } t \geq 0
$$

Therefore,

$$
\begin{array}{ll}
\frac{d}{d t}\left(C_{d}(t)-M C_{i}(t)\right)=0 & \text { for all } t \geq 0 \\
C_{d}(t)=M C_{i}(t)+T & \text { for all } t \geq 0
\end{array}
$$

where $T$ is the sum of the initial concentrations of independent species. Based on the above equation, the ordinary differential equations for the kinetic equations can be eliminated from the computation. Finally, the reduced model is obtained in the differential equation form.

$$
\frac{d}{d t} C_{i}(t)=S_{R} r(C(t), k)=S_{R} r\left(\left[C_{i}(t), M C_{i}(t)+T\right], k\right) \quad \text { for all } t \geq 0
$$

\subsection{Steady-state Determination}

The steady-states can be identified by the Newton-Raphson method. This algorithm is a robust method for solving the nonlinear equations. In this method, the derivative of an equation, and extrapolates along the derivative are calculated until the solution comes to satisfy the given tolerance. At a steady-state,

$$
\frac{d C_{i}(t)}{d t}=S_{R} r(C(t), k)=0
$$

In the $k$ th iteration, the next solution is evaluated as

$$
r_{k+1}=r_{k}-J^{-1}\left(r_{k}\right) S_{R} r_{k}
$$

where $r_{k}$ is the reaction rate at the $k$ th iteration, and $J$ is the Jacobian matrix of $r_{k}$. The iteration stops as soon as the requested tolerance is achieved.

$$
\left\|S_{R} r_{k+1}\right\|-\left\|S_{R} r_{k}\right\|<\text { tol }
$$

where tol is the desired tolerance. For the systems without the steady-states, however, the algorithm displays the concentration at the time limit as the solution. Therefore, the stability of the obtained steady-states should be inspected to check whether the result indicates the real steady-state or not.
The kinetic models are further reduced by differentiating their time scales. The automated complexity reduction for all possible dynamics of biological systems can be conducted with Low-Dimensional Manifolds (Zobeley et al., 2005). Using the quasi-steady-state approximation, the reactions that are substantially faster than the reaction of our interest are neglected from the kinetic processes.

\subsection{Stability Analysis of the Obtained Steady-state}

Stability analysis investigates whether a system always returns to the reference state after a perturbation. The dynamic behaviours of most biochemical systems can be modelled as a set of nonlinear differential equations. These dynamic behaviours can be analyzed by linearizing the nonlinear system.

For the steady-state concentration $C_{i, s}$,

$r\left(C_{i, s s}\right)=0$

The above nonlinear differential equation can be expanded in a Taylor series about $C_{i, s s}$.

$r\left(C_{i}\right)=r\left(C_{i, s s}\right)+r^{1}\left(C_{i, s s}\right)\left(C_{i}-C_{i, s s}\right)+\frac{1}{2} r^{2}\left(C_{i, s s}\right)\left(C_{i}-C_{i, s s}\right)^{2}+\cdots$

The high-order terms than the second order in the Taylor series expansion can be ignored. The above equation can be rearranged as,

$r\left(C_{i}\right)=J \zeta$

where

$\zeta=C_{i}-C_{i, s s}$

To prove the stability of a nonlinear system, the inclusion of decaying exponentials should be checked. For the eigenvalue lambda of the Jacobian matrix $J$,

$J \zeta=\lambda \zeta$

Each eigenvalue is paired with the corresponding eigenvector. When the zeta is not zero, vector solutions are the eigenvectors of $J$. The characteristic equation of the Jacobian matrix $\mathbf{J}$ is

\subsection{Time Scale Analysis}




$$
[J-\lambda \mathrm{I}] \zeta=\left|\begin{array}{cccc}
j_{11}-\lambda & j_{12} & \cdots & j_{1 n} \\
j_{21} & j_{22}-\lambda & \cdots & j_{2 n} \\
\vdots & \vdots & \vdots & \vdots \\
j_{n 1} & j_{n 2}-\lambda & \cdots & j_{n n}-\lambda
\end{array}\right|=0
$$

For stability analysis, the above equation is extended to the $n$th order equation in lambda.

$$
\operatorname{det}(J-\lambda I)=\alpha_{0} \lambda^{n}+\alpha_{1} \lambda^{n-1}+\ldots+\alpha_{n-1} \lambda^{1}+\alpha_{0}=0
$$

The stability can be determined referring this eigenvalue.

\subsection{Calculation of the Control Coefficients}

For the systems with steady-states or sustained oscillatory behaviours, the corresponding control coefficients are calculated as illustrated in section 2. For the other systems, and for the transient period before reaching the steady-state or sustained oscillation, the time-varying control coefficients are obtained.

\section{ILLUSTRATION}

The proposed approach is demonstrated with the kinetic model of ERK pathway developed by Cho et al. (2003). For this model, 4 conservation relations are identified with WebCell (Lee et al., 2006) as follow.

$\mathrm{RP}+\mathrm{RKIP}-\mathrm{P} / \mathrm{PP}=163.194 \mathrm{e}-6 \mathrm{M}$

RAF-1*/RKIP/ERK-PP + ERK-P + MEK-PP/ERK + ERK$\mathrm{PP}=259.918 \mathrm{e}-6 \mathrm{M}$

Raf-1*+ RAF-1*/RKIP + RAF-1*/RKIP/ERK-PP = 126.291 e-6 M

RKIP + RAF-1*/RKIP + RAF-1*/RKIP/ERK-PP + RKIP-P + MEK-PP + MEK-PP/ERK + RKIP-P/PP $=152.685 \mathrm{e}-6 \mathrm{M}$

The steady-state concentrations are obtained as shown in Table 1 .

\section{Table 1. Steady-state concentrations of ERK} pathway

\begin{tabular}{|c|c|c|}
\hline No & Compounds & Concentration $(* \mathrm{e}-6 \mathrm{M})$ \\
\hline 1 & $R a f-1^{*}$ & 67.3 \\
\hline 2 & $R K I P$ & 0.0521 \\
\hline 3 & $R A F-1^{*} / R K I P$ & 0.0183 \\
\hline 4 & $R A F-1^{*}$ & 59.0 \\
\hline 5 & /RKIP/ERK-PP & 0.0393 \\
\hline 6 & RKKIP-P $P$ & 0.0126 \\
\hline 7 & $M E K-P P$ & 65.4 \\
\hline
\end{tabular}

\begin{tabular}{|c|c|c|}
\hline 8 & $M E K-P P / E R K$ & 26.2 \\
\hline 9 & $E R K-P P$ & 175 \\
\hline 10 & $R P$ & 161 \\
\hline 11 & $R K I P-P / P P$ & 2.14 \\
\hline
\end{tabular}

Subsequently, time scale analysis (Zobeley et al., 2005) using Matlab substitutes 2 out of remaining 7 differential equations with 2 algebraic equations. Stability analysis showed that these steady-states are all stable.

Finally, the time-varying control coefficients are obtained for the initial transient states and for the steady-states. For example, the effects of parameter $\mathrm{k} 5$ on ERK-P and ERK-PP are described in Figs. 2 and 3.

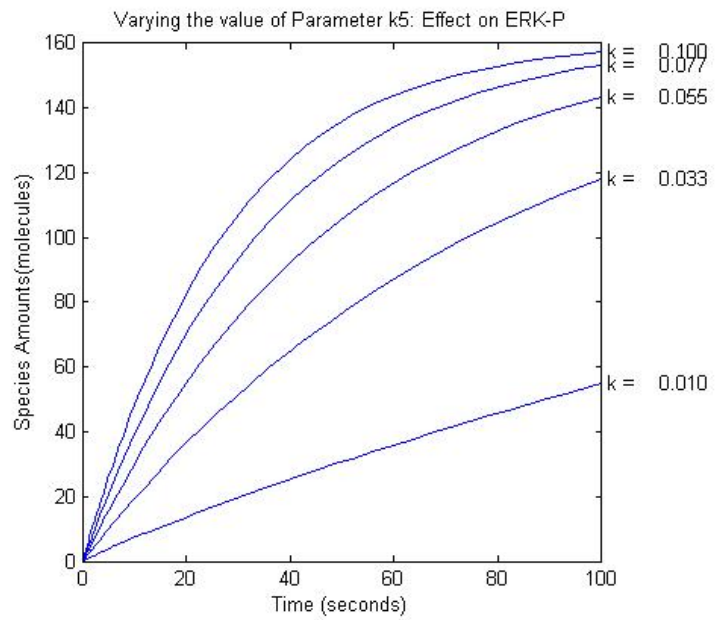

Fig. 2. The effect of the change in the value of $\mathrm{k} 5$ on ERK-P.

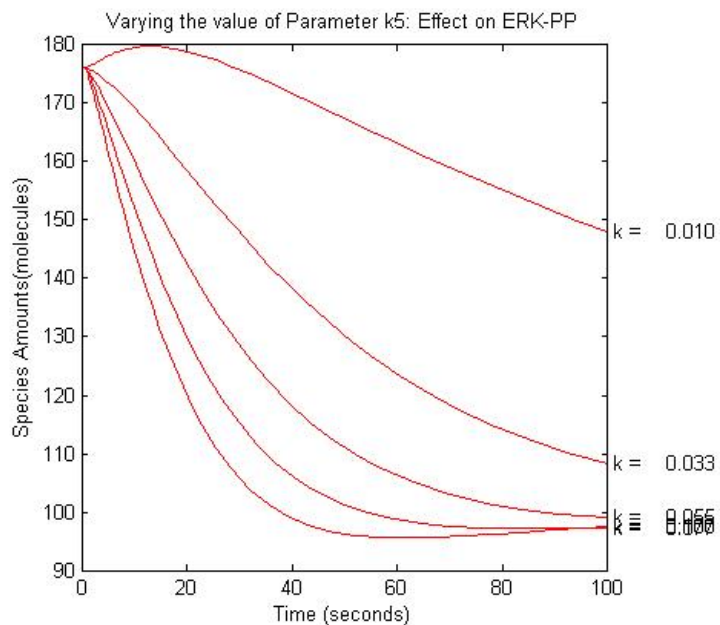

Fig. 3. The effect of the change in the value of $\mathrm{k} 5$ on ERK-PP. 


\section{CONCLUSIONS}

A systematic approach for the metabolic control analysis has been proposed to this end. The complex biological systems are reduced by identifying the conservation relations and the time scale analysis. The control coefficients for the reduced models are classified into the three groups based on their stabilities at the steady-states. The proposed approach has been illustrated with its application to a signaling pathway.

\section{ACKNOWLEDGEMENT}

Financial support for this work was received from the Korean Systems Biology Research Program (M1030902000003B5002-00000) under the auspices of the Ministry of Science and Technology through the Korea Science and Engineering Foundation as well as the BK21 project; LG Chem (Chair Professorship for S.Y. Lee), Microsoft and IBM-SUR program.

\section{REFERENCES}

Acerenza, L., H.M. Sauro and H. Kacser (1989). Control analysis of time-dependent metabolic systems. Journal of Theoretical Biology, 151, 423-444.

Cho, K.-H., S.-Y Shin, H.-W. Kim, O. Wolkenhauer, B. McFerran and W. Kolch (2003). Mathematical modeling of the influence of RKIP on the ERK signaling pathway, CSMB 2003, 127-141.

Ghosh, A. and B. Chance (1964). Oscillations of glycolytic intermediates in yeast cells. Biochemical and Biophysical Research Communications, 16, 174-181.

Heinrich, R. and T.A. Rapoport (1974a). A linear steady-state treatment of enzymatic chains - critique of the crossover theorem and a general procedure to identify interaction sites with an effector. European Journal of Biochemistry, 42, 97-105.

Heinrich, R. and T.A. Rapoport (1974b). A linear steadystate treatment of enzymatic chains - general properties, control and effector strengths. European Journal of Biochemistry, 42, 89-95.

$\mathrm{Hu}, \mathrm{D}$. and J.M. Yuan (2006). Time-dependent sensitivity analysis of biological networks: coupled MAPK and PI3K signal transduction pathways. Journal of Physical Chemistry A, 110, 5361-5370.

Johnson, H.E, D. Broadhurst, D.B. Kell, M.K. Theodorou, R.J. Merry and G.W. Griffith (2004). High-throughput metabolic fingerprinting of legume silage fermentations via Fourier transform infrared spectroscopy and chemometrics. Applied and Environmental Microbiology, 70, 1583-1592.

Kacser, H. and J.A. Burns (1973). The control of flux. Symposium of the Society for Experimental Biology, 27, 65-104.

Lee, D.-Y., C. Yun, A. Cho, B.K. Hou, S. Park, and S.Y. Lee (2006). WebCell: a web-based environment for kinetic modeling and dynamic simulation of cellular networks. Bioinformatics, 22, 1150-1151.

Reijenga, K.A., H.V. Westerhoff, B.N. Kholodenko and J.L.
Snoep (2002). Control analysis for autonomously oscillating biochemical networks. Biophysical Journal, 82, 99-108.

Villas-Boas, S.G., J.F. Moxley, M. Akesson, G. Stephanopoulos and J. Nielsen (2005). High-throughput metabolic state analysis: the missing link in integrated functional genomics of yeasts. Biochemisry Journal, 388, 669-677.

Zobeley, J., D. Lebiedz, J. Kammerer, A. Ishimurzin and U. Kummer (2005). A New Time-Dependent Complexity Reduction Method for Biochemical Systems. In Transactions on Computational Systems Biology, C. Praiami et al (Eds.), 3380, 90-110. SpringerVerlag Berlin Heidelberg. 